\title{
Relationship between the Depth of Penetration and Fracture Resistance of Various Sealers: A Comparative Study
}

Abhijeet K Kakani ${ }^{1}$, Chandrasekhar Veeramachaneni ${ }^{2}$, Muralidhar Tummala ${ }^{3}$, Chandrakanth Majeti ${ }^{4}$, Ravichandra Ravi ${ }^{5}$, Wasifoddin A Chaudhari ${ }^{6}$

\begin{abstract}
Aim: The aim of this in vitro study was to estimate the penetration depth and fracture resistance of three different sealers and to verify the relationship between the penetration depth and fracture resistance.

Materials and methods: Sixty single-rooted teeth were selected and root canal preparation was done. After the instrumentation, the teeth were divided into three groups of 20 each. The groups were then obturated with gutta-percha (GP)-AH Plus sealer, Resilon-Real Seal, and propointbioceramic sealers, respectively. Ten teeth from each group were sectioned at three different regions (i.e., coronal, middle, and apical thirds of the root canal) and were viewed under a confocal microscope to determine the penetration depth of the sealer. The remaining samples were subjected to fracture resistance under a universal testing machine and the statistical analysis was done by using one-way ANOVA and post hoc Bonferroni tests.

Results: Propoint-bioceramic group showed the highest fracture resistance values followed by GP-AH Plus sealer and Resilon-Real seal groups with no significant difference noticed between them. Depth of penetration was greater for GP-AH Plus sealer, propoint-bioceramic, with no significant difference followed by the Resilon-Real seal group.

Conclusion:The newer obturating material propoint-bioceramic group showed a greater fracture resistance. No correlation could be established between the depth of sealer penetration and fracture resistance.

Clinical significance: Fracture resistance of tooth obturated with propoint-bioceramic sealer combination is significantly greater than GP-AH Plus and Resilon-Real seal combination, thereby showing propoint-bioceramic as a promising obturating material.

Keywords: Bioceramic sealers, Confocal laser scanning microscopy, Fracture resistance, Penetration depth, Universal testing machine.

The Journal of Contemporary Dental Practice (2021): 10.5005/jp-journals-10024-3038
\end{abstract}

\section{INTRODUCTION}

Microbes and their byproducts are the main culprits implicated in the pulpal and associated periapical problems. The main aim of the endodontic treatment is to debride the root canal system devoid of microorganisms. The anatomical complexity of the root canals (lateral canals and tubules) highlights the existence of microorganisms in root canals that offer protection to microorganisms from the antibacterial actions of various disinfectants. Therefore, complete debridement of the root canal system is a key step in managing the infected root canals and providing a fluid-tight seal using a biocompatible obturating material. ${ }^{2}$

The complete three-dimensional (3D) obturation provides a hermetic seal to the root canal system closing all the possible avenues of leakage from the oral cavity and periradicular tissues. ${ }^{3,4}$

A combination of core material and sealer is the most common procedure used for obturation. The advantage of using a sealer is well known; it creates a union between the core material and the canal wall by sealing off any residual spaces; it can penetrate the accessory and lateral canals, and the dentinal tubules. ${ }^{5,6}$ Because of the well-known potential of the bacteria to colonize dentinal tubules the deeper penetration of a sealer is advantageous to show their antibacterial effects and to cause the viable bacterial entombment, thereby depriving them of potential nutrient sources. ${ }^{4-8}$

One of the potential complications after endodontic therapy is root fracture. The factors responsible for postendodontic root fracture include loss of the tooth structure, stresses induced by cavity preparation, instrumentation, obturation, irrigation, coronal

\begin{abstract}
1,6 Department of Conservative Dentistry and Endodontics, Nanded Rural Dental College and Research Center, Nanded, Maharashtra, India

${ }^{2}$ Department of Conservative Dentistry and Endodontics, Mallareddy Dental College for Women, Hyderabad, Telangana, India

${ }^{3}$ Department of Conservative Dentistry and Endodontics, Mamata Dental College, Khammam, Telangana, India

${ }^{4}$ Department of Conservative Dentistry and Endodontics, Army Dental College and Hospital, Hyderabad, Telangana, India

${ }^{5}$ Department of Conservative Dentistry and Endodontics, GITAM Dental College and Hospital, Visakhapatnam, Andhra Pradesh, India

Corresponding Author: Ravichandra Ravi, Department of Conservative Dentistry and Endodontics, GITAM Dental College and Hospital, Visakhapatnam, Andhra Pradesh, India, Phone: +0 91-9155717777, e-mail: raavi.ravi13@gmail.com
\end{abstract}

How to cite this article: Kakani AK, Veeramachaneni C, Tummala M, et al. Relationship between the Depth of Penetration and Fracture Resistance of Various Sealers: A Comparative Study. J Contemp Dent Pract 2021;22(1):34-38.

Source of support: Nil

Conflict of interest: None

restoration, and inappropriate selection of tooth abutments for prosthesis. ${ }^{9}$

The depth of penetration of sealers mainly depends on the variations in physical and chemical properties. ${ }^{10}$ Therefore, a 
comparison of the penetration ability of various root canal sealers that are routinely used in clinical practice becomes important. Hence, the objective of the current study was to evaluate the depth of penetration of three different sealers by using a confocal laser scanning microscope (CLSM) and determine root fracture resistance by using a universal testing machine.

\section{Materials and Methods}

\section{Sample Selection and Preparation}

The research protocol of this in vitro study was approved by the research committee, and the ethical clearance was obtained from the Ethics Committee of Nanded Rural Dental College and Research Center, Nanded. In the current study, 60 single-rooted mandibular premolars that were extracted due to orthodontic reasons were collected; prior informed consent was obtained from the patients, and the teeth samples were stored in sterile saline $(0.9 \% \mathrm{NaCl})$ solution until further use. The presence of a single root canal and also any previous root canal treatment in each tooth were confirmed by obtaining the radiographs in the buccolingual and mesiodistal directions. Each tooth below the cementoenamel junction (CEJ) was decoronated using safe sided diamond disc (IsoMet, Buehler Ltd., Lake Bluff, Illinois, USA) underwater coolant to a standard length of $12 \mathrm{~mm}$. Foraminal patency was confirmed with a No.10 K-file, and the root canals were enlarged using Protaper endodontic rotary instruments (Dentsply Maillefer, Ballaigues, Switzerland) until file F3 reached the working length ( $1 \mathrm{~mm}$ from apical foramen). Irrigation between the instruments was performed using 3\% sodium hypochlorite ( $\mathrm{NaOCl}$ ) (Vishal Dentocare Pvt. Ltd, Ahmedabad, India). The final active rinse was done using $17 \%$ ethylenediaminetetraacetic acid (EDTA) (Canalarge, Ammdent, Chandigarh, India) solution for removal of smear layer followed by a distilled water rinse. All the irrigants were delivered using a 27-gauge needle placed as far into the canal as possible without binding, and the canals were dried using paper points of size 30 (6\% taper) (Pearl Dent Co. Ltd., Hochiminh, Vietnam).

\section{Sample Preparation for Fracture Resistance}

Half of the above specimens ( $n=30$ ) were subjected to fracture resistance and divided into three groups (G I, II, III, each $n=10$ ) based on the obturating material used to fill the root canal.

Group I: The root canals were filled with Resilon using a Real seal (SybronEndo, Orange, California, USA) as the sealer. Real seal (SybronEndo) primer was applied first with a micro brush and left for 30 seconds, and excess primer was removed using paper points of size 30 (6\% taper). The Real seal sealer was placed with a lentulo spiral in the canal and obturated with tip size 30 (6\% taper) Resilon cone. The excess material was sheared off with a plugger $1 \mathrm{~mm}$ below the canal orifice and then the material was cured in the root canal with LED light (Bluephase C8, Ivoclar Vivadent, Schaan, Liechtenstein) for 30 seconds

Group II: Root canals were filled with propoint (DRFP Ltd., Stamford, UK) using a bioceramic sealer Smartpaste Bio (Pro smart-DRFP Ltd., Stamford, UK). Bioceramic sealer was placed with a lentulo spiral in the canal and obturated with No.30 (6\% taper) propoint. The excess material was removed using smart trim burs (DRFP Ltd., Stamford, UK) without water.

Group III: Root canals in this group were filled with gutta-percha (Diadent Group International, BC, Canada) using AH Plus (Dentsply
International Inc., Germany) as the sealer. The $\mathrm{AH}$ - Plus sealer was applied to the root canal using a lentulo spiral and obturating with a No.30 (6\% taper) gutta-percha (GP) cone. The excess material was sheared off with a plugger $1 \mathrm{~mm}$ below the canal orifice.

All the specimens were stored in an incubator (Yorco sales Pvt. Ltd., New Delhi) at $37^{\circ} \mathrm{C}$ in $100 \%$ humidity for 72 hours to allow the setting of the sealer. Each specimen was wrapped in aluminum foil and embedded in acrylic resin placed in a plastic mold. After 24 hours, the specimens were removed from the set acrylic mold, the foil was peeled off, and space was then replaced using polyvinyl siloxane (PVS) elastomeric impression material (3M ESPE, Seefeld, Germany) to simulate the periodontal ligament. Excess PVS was removed using No.12 Bard-Parker blade (Kiato Surgical Blades, Gurgaon, Haryana, India). All the specimens were stored in an incubator at $37^{\circ} \mathrm{C}$ in $100 \%$ humidity for one week (Yorco sales Pvt. Ltd., New Delhi).

Fracture resistance was then evaluated under a universal testing machine (Autograph AG 15, Shimadzu, Kyoto, Japan). A custom-made jig (tip diameter: $1 \mathrm{~mm}$ ) was used to maintain or limit its contact only with the obturating material. The jig was driven downward at a speed of $10 \mathrm{~mm} / \mathrm{min}$ into the filling material until the root fractured. The force required for fracture was recorded in newton (N) for each root in case of an instantaneous drop of applied force greater than $25 \%$ of the applied force.

\section{Sample Preparation for Confocal Laser Scanning Microscopic Analysis}

The other half of the specimens $(n=30)$ were used to determine the depth of sealer penetration. They were divided into three groups ( $n=10$ each) based on the obturating material used to fill the root canal. The procedure for obturation and storage in each group was similar to groups I, II, III, respectively, as done for fracture resistance. The difference is Rhodamine B fluorescent dye (Chennai Chemicals, Chennai, India) was added to the sealer to observe the specimens under a confocal microscope.

After the storage period, two markings were made on each specimen at the junction of coronal and middle thirds, middle, and apical thirds of the root canal. A hard-tissue microtome was used to section the root horizontally into three segments with a standard size of $2 \mathrm{~mm}$ each by reducing it coronally and apically by using a series of silicon carbide disks (3M ESPE, Seefeld, Germany).

The sections obtained were then observed under a confocal microscope (Carl Zeiss, Germany), and the depth of sealer penetration was assessed using LSM image browser software.

\section{Statistical Analysis}

The data obtained for fracture resistance and depth of sealer penetration were subjected to statistical analysis using one-way ANOVA and post hoc Bonferroni tests.

\section{Results}

\section{Group Comparison for Fracture Resistance}

Table 1 displays the mean fracture resistance values of all the groups. One-way ANOVA indicated a significant difference between G II and the other two groups ( $p<0.05$ ) with higher values for G II. However, no statistically significant difference between $\mathrm{G} I$ and $\mathrm{G}$ III $(p>0.05)$ was noticed.

Table 2 displays the mean depths of sealer penetration of all the groups at various root regions. When compared statistically, a significant difference was observed between the groups I, II*, 
Depth of Penetration and Fracture Resistance of Sealers

Table 1: Mean fracture resistance values of all the groups

\begin{tabular}{llll}
\hline & Coronal & Middle & Apical \\
Group & Mean $\pm S D$ & Mean $\pm S D$ & Mean $\pm S D$ \\
\hline Resilon + real seal & $862.76 \pm 188.66^{\mathrm{a}}$ & $605.78 \pm 71.90^{\mathrm{a}}$ & $221.85 \pm 70.79^{\mathrm{a}}$ \\
AH Plus + gutta-percha & $1217.91 \pm 170.96^{\mathrm{b}}$ & $947.57 \pm 128.03^{\mathrm{b}}$ & $369.99 \pm 99.92^{\mathrm{b}}$ \\
Propoint + bioceramic sealer & $1148.32 \pm 108.21^{\mathrm{bc}}$ & $937.85 \pm 124.99^{\mathrm{bc}}$ & $301.81 \pm 69.39^{\mathrm{ab}}$ \\
$p$-value & $<0.001^{*}$ & $<0.001^{*}$ & $<0.001^{*}$ \\
\hline
\end{tabular}

${ }^{*} p<0.001$; highly significant at $1 \%$ level; SD, standard deviation; Different alphabets show significant differences among the three groups

Table 2: Mean depths of sealer penetration of all the groups at various root regions

\begin{tabular}{llllll}
\hline Group & $N$ & Mean & Std. deviation & Minimum & Maximum \\
\hline AH Plus + GP & 10 & 153.4490 & 9.39737 & 140.08 & 168.81 \\
$\begin{array}{l}\text { Resilon + real } \\
\text { seal }\end{array}$ & 10 & 160.5250 & 8.43658 & 150.56 & 175.90 \\
$\begin{array}{l}\text { Propoint + } \\
\text { bioceramic }\end{array}$ & 10 & 232.4690 & 9.97453 & 219.17 & 248.44 \\
sealer & & & & \\
\hline
\end{tabular}

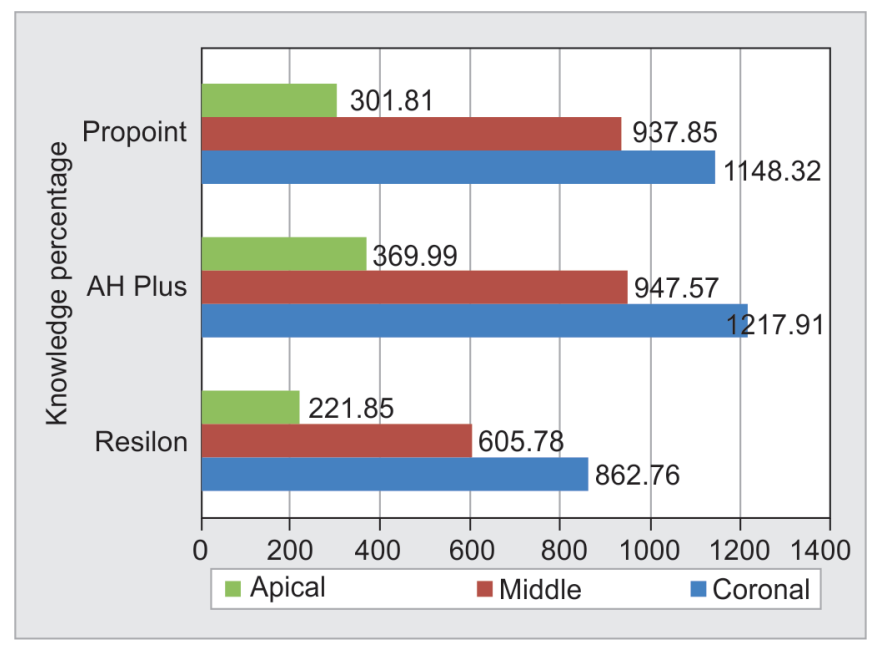

Fig. 1: Penetration of sealers at coronal, middle, and apical levels

and III* (greater values for groups are marked with an asterisk, $p<0.001)$. This indicated that Resilon-real seal, in comparison with propoint-bioceramic and GP-AH Plus was associated with the least sealer penetration depth. Also, statistically significant differences were observed between coronal, middle, and apical sections with a greater depth of penetration for coronal followed by middle then apical sections, respectively (Figs 1 and 2A to C).

\section{Discussion}

One of the weakest links in endodontic therapy is obturating techniques. Materials that use $50 \%$ of an obturated tooth are challenged by bacterial penetration through the entire length of the canal within 30 days. ${ }^{11}$ Therefore, improving the obturating materials and techniques over the traditional ones might enhance the quality of treatment outcomes and prevent chances of reinfection by entombment of remaining bacteria. ${ }^{12,13}$
It is well established that the endodontic treatment changes the mechanical properties of the teeth, and the fatigue failures might result due to increased functional and para-functional stresses. ${ }^{13}$

To date, there is no consensus on the best obturation material in endodontics. To some extent, GP-AH Plus obturation is considered to be the gold standard in endodontics. But now other bioceramic sealers are gaining popularity and significance. Also, the propoint is claimed to have a hydrophilic property and expands after obturation forming monoblock, and causes root reinforcement. Resilon-Real seal also gained popularity using the monoblock concept, so the present study aimed to compare these materials to suggest a better obturating material-sealer combination. Though several studies in the literature have correlated the penetration of various sealers and fracture resistance, the present study was undertaken as there are very few studies that compare the combinations used in our study. Though the results correlate with the depth of sealer penetration and fracture resistance, previous studies show no conclusive result; hence, ample scope exists in this horizon to address these concerns.

In recent years, novel obturating and hydrophilic materials, that expand upon absorption of water to create a fluid-tight impermeable seal, have been introduced. ${ }^{12-14}$ Propoint also known as C-point, a hydrophilic material, expands in the presence of moisture. ${ }^{14}$ So this property of the material assures a fluid-tight seal in the root canal and enhances root reinforcement. Results of the current study indicate the positive outcome of propoint, including its highest fracture resistance values than those of the other two groups. Also, there was no statistically significant difference observed between GP-AH Plus and Resilon-real seal groups.

Mandibular premolars were specially selected because of their delicate tooth morphology and the functional stresses that extend into them replicate the clinical condition where the chewing forces exerted are maximum. ${ }^{2}$

A lot of debate exists in the literature regarding the root reinforcement provided by GP-AH Plus and Resilon-Real seal. Few studies have concluded that the GP-AH Plus combination has greater fracture resistance than Resilon-Real seal ${ }^{15,16}$ and viceversa. ${ }^{9,17}$ Interestingly, one study showed no significant difference between both the groups; ${ }^{18}$ results of the present study are similar to the findings in that study. Authors in support of the GP-AH Plus combination states that Resilon is not stiff enough to provide a mechanically homogenous unit with root dentin as is provided by GP. Also, the authors in support of the Resilon-Real seal state that it has a greater depth of sealer penetration and greater contact with the canal wall enhances the fracture resistance.

Lertchirakarn et al. highlighted that sealer, penetration was proportional to fracture resistance ${ }^{19}$ but the findings of the current study establish no correlation between both factors. Though GP-AH Plus showed deeper sealer penetration, it has less fracture resistance than the propoint-bioceramic group. This is in accordance with 

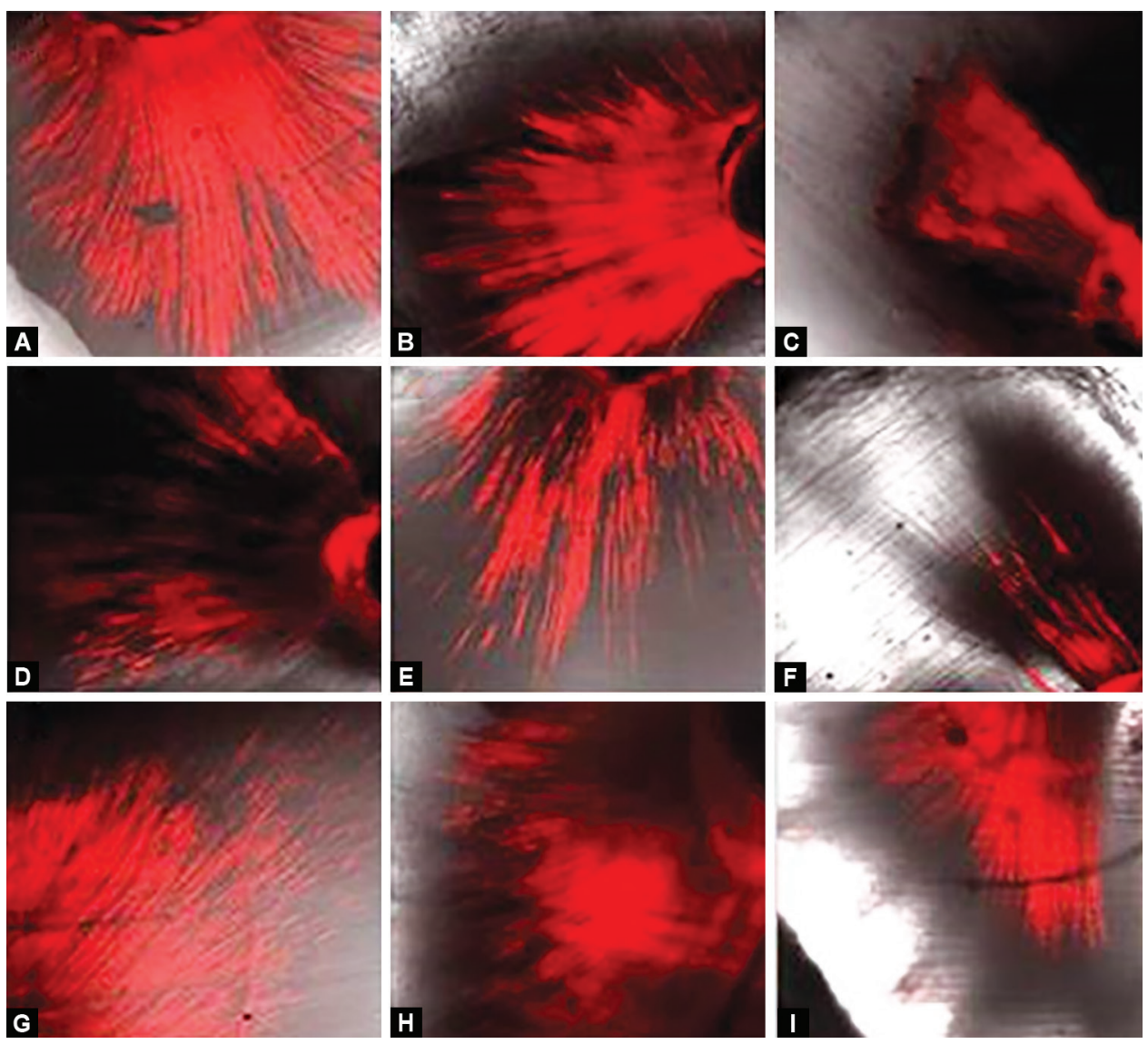

Figs. 2: Confocal microscopic images (10x): depth of penetration of sealers at coronal, middle, and apical levels: (A-C) Bioceramic, (D-F) Real seal, and (C-I) AH Plus

the study conducted by Arikatla et al. where the AH-Plus sealer has shown a higher depth of penetration with minimal gaps when compared to the bioceramic sealers, this may be due to a higher concentration of epoxy resin. Superior adaptation with minimal gaps might be due to its property of bonding chemically to root dentin by creating covalent bonds between the collagen and the epoxy resin. ${ }^{20}$

Also, the least depth of penetration was observed in the Resilon-real seal group, but there was no significant difference in the fracture resistance values when compared with GP-AH plus group.

The probable reason for greater fracture resistance values for the propoint group might be the expansion of core material and attaining a homogenous monoblock. The reason for the greater depth of sealer penetration in AH-Plus and bioceramic groups might be the longer setting time compared to the Resilon-real seal group which leads to greater capillary action, thereby providing a better seal between the walls of root canals and the core material. ${ }^{19}$

Yendrembam et al. ${ }^{21}$ argued that the highest fracture resistance was observed with the bioceramic sealer when compared with the AH-Plus sealer and MTA fill apex. The reason cited for the above finding was that the bioceramic sealer can produce hydroxyapatite throughout the setting mechanism. Due to this, the bioceramic sealers form a bond in the presence of dentine. Another advantage of bioceramic sealer is its low contact angle that allows easy spread throughout the canal.

Contrary to the above findings, Celikten et al. highlighted that the bioceramic sealers had shown significantly lower values for a fracture than the control group sealers. They also stated that the root canal sealers which are based on bioceramic or calcium silicate, require water for the setting process and these materials use the moisture within the dentinal tubules to initiate and complete the setting mechanism. So the moisture present in the tubules may not be sufficient for the setting mechanism of these materials, leading to the lower resistance to fracture of the root that was obturated with bioceramic sealers. ${ }^{22}$

We used a CLSM to evaluate the depth of penetration. Picoh et al. reported that the artifacts could practically be excluded using CLSM. ${ }^{23}$ Other benefits of CLSM include determining precise field depth, eliminating background information away from the focal plane, and capability of making optical sections even from thick specimens. ${ }^{7,24}$

There is a remarkable difference in the depth of sealer penetration at various root regions, with the coronal region showing the greater depth of penetration followed by middle and apical in all the groups. This finding corroborates findings of previous studies, which are attributed to reasons like a decrease in the density of dentinal tubule from coronal to apical region, ${ }^{25-28}$ inability to completely remove the smear layer in apical areas that interfere with sealer penetration. ${ }^{4,20}$

\section{Limitations of the Study}

- It is an in vitro study and results may be different under in vivo conditions. 
- Single cone techniques were employed in the current study, but these techniques need to be confirmed by comparing them with other obturating techniques (e.g., lateral condensation, warm vertical).

\section{Conclusion}

Overall, the findings of the current study reveal that the new combination of the propoint-BC sealer showed greater fracture resistance than GP-AH Plus and Resilon-Epiphany. Propoint-BC sealer combination is a better alternative than other sealers in terms of root reinforcement. Also, a sealer penetration does not reinforce and enhance the fracture resistance but the core material-sealer adhesion and/or expansion reinforces the tooth fracture resistance. Further in-vitro and in vivo studies are needed to support the results of the present study.

\section{References}

1. Kakehashi S, Stanley HR, Fitzgerald RJ. The effects of surgical exposures of dental pulps in germ-free and conventional laboratory rats. Oral Surg Oral Med Oral Pathol 1965;20(3):340-349. DOI: 10.1016/0030-4220(65)90166-0.

2. Ravi RC, Mandava J, Chalasani U, et al. M. Influence of storage time and cementation strategies on push-out bond strength of fiber posts to root dentin. J Clin Diagn Res 2017;11(12):ZC05-ZC08. DOI: 10.7860/ JCDR/2017/29866.10924.

3. Arora S, Hegde V. Comparative evaluation of a novel smart-seal obturating system and its homogeneity of using cone-beam computed tomography: in vitro simulated lateral canal study. J Conserv Dent 2014;17(4):364-368. DOI: 10.4103/0972-0707.136512.

4. Mamootil K, Messer HH. Penetration of dentinal tubules by endodontic sealer cements in extracted teeth and in vivo. Int Endod J 2007;40(11):873-881. DOI: 10.1111/j.1365-2591.2007.01307.x.

5. Gutmann JL, Witherspoon DE. Obturation of the cleaned and shaped root canal system. In: Cohen S, Burns R, eds. Pathways of the Pulp, 8th ed. St Louis, MO: CV Mosby; 2004. pp. 293-364.

6. Hata G, Kawazoe S, Toda T, et al. Sealing ability of Thermafil with and without sealer. J Endod 1992;18(7):322-326. DOI: 10.1016/S00992399(06)80481-4.

7. Patel DV, Sherriff M, Ford TRP, et al. The penetration of RealSeal primer and Tubliseal into root canal dentinal tubules: a confocal microscopic study. Int Endod J 2007;40(1):67-71. DOI: 10.1111/j.13652591.2006.01184.x.

8. Heling I, Chandler NP. The antimicrobial effect within dentinal tubules of four root canal sealers. J Endod 1996;22(5):257-259. DOI: 10.1016/ S0099-2399(06)80144-5.

9. Jeanne M, Ataide N, Paul C, et al. In vitro resistance to fracture of roots obturated with Resilon or gutta-percha. J Endod 2011;37(6):828-831. DOI. 10.1016/j.joen.2011.02.024.

10. Oskan T, Aktener $\mathrm{O}$, Sen $\mathrm{BH}$, et al. The penetration of root canal sealers into dentinal tubules: a scanning electron microscopic study. Int Endod J 1993;26(5):301-305. DOI: 10.1111/j.1365-2591.1993.tb00575.x.

11. Torabinejad M, Ung B, Kettering JD. In vitro bacterial penetration of coronally unsealed endodontically treated teeth. J Endod 1990;16(12):566-569. Doi:10.1016/S0099-2399(07)80198-1.
12. Majeti $C$, Veeramachineni $C$, Ravi R. Effect of silanization on pushout bond strength of $\mathrm{H} 2 \mathrm{O} 2$-etched fiber posts using various resin cements: an in vitro study. J Oper Dent Endod 2019;4(1):27-32. DOI: 10.5005/jp-journals-10047-0071.

13. Mandava J, Chang PC, Roopesh B, et al. Comparative evaluation of fracture resistance of root dentin to resin sealers and an MTA sealer: An in vitro study. J Conserv Dent 2014;17(1):53-56. DOI: 10.4103\%2F0972-0707.124140.

14. Didato A, Eid AA, Levin MD, et al. Time-based lateral hygroscopic expansion of a water-expandable endodontic obturation point. J Dent 2013;41(9):796-801. DOI: 10.1016/j.jdent.2013.06.012.

15. Karapinar Kazandag M, Sunay H, Tanalp J, et al. Fracture resistance of roots using different canal filling systems. Int Endod J 2009;42(8):705710. DOI:10.1111/j.1365-2591.2009.01571.x.

16. Lertchirakarn V, Poonkaew A, Messer H. Fracture resistance of roots filled with Gutta-percha or Realseal ${ }^{\circledR}$. Int Endod J 2011;44(11):10051010. DOI: 10.1111/j.1365-2591.2011.01908.x.

17. Hammad M, Qualtrough A, Silikas N. Effect of New Obturating Materials on Vertical Root Fracture Resistance of Endodontically Treated Teeth. J Endod 2007;33(6):732-736. DOI: 10.1016/j.joen. 2007.02.004.

18. Ersev H, Yilmaz B, Pehlivanoğlu E, et al. Resistance to vertical root fracture of endodontically treated teeth with MetaSEAL. J Endod 2012;38(5):653-656. DOI: 10.1016/j.joen.2012.02.015.

19. Lertchirakarn V, Timayam A, Messer HH. Effects of root canal sealers on vertical root fracture resistance of endodontically treated teeth. J Endod 2002;28(3):217-219. DOI: 10.1097/00004770-200203000-00018.

20. Arikatla SK, Chalasani U, Mandava J, et al. Interfacial adaptation and penetration depth of bioceramic endodontic sealers. J Conserv Dent 2018;21(4):373-377. DOI: 10.4103/JCD.JCD_64_18.

21. Yendrembam B, Mittal A, Sharma N, et al. Relative assessment of fracture resistance of endodontically treated teeth with epoxy resin-based sealers, AH Plus, MTA Fillapex, and Bioceramic Sealer: an in vitro study. Indian J Dent Sci 2019;11(1):46-50. DOI: 10.4103/IJDS. IJDS_45_18.

22. Celikten B, Uzuntas CF, Gulsahi K. Resistance to fracture of dental roots obturated with different materials. Biomed Res Int 2015;2015:591031. DOI: 10.1155/2015/591031.

23. Marissa C, Usman M, Suprastiwi E, et al. Comparison of dentinal tubular penetration of three bioceramic sealers. Int J App Pharm 2020;12(2):23-26. DOI: 10.22159/ijap.2020.v12s2.OP-05.

24. Gharib SR, Tordik PA, Imamura GM, et al. A confocal laser scanning microscope investigation of the epiphany obturation system. J Endod 2007;33(8):957-961. DOI: 10.1016/j.joen.2007.03.011.

25. Ravi SV, Nageswar R, Swapna H, et al. Epiphany sealer penetration into dentinal tubules: confocal laser scanning microscopic study. J Conserv Dent 2014;17(2):179-182. DOI: 10.4103/0972-0707.128056.

26. Mjor IA, Smith MR, Ferrari $M$, et al. The structure of dentine in the apical region of human teeth. Int Endod J 2001;34(5):346-353. DOI: 10.1046/j.1365-2591.2001.00393.x.

27. Carrigan PJ, Morse DR, Furst ML, et al. A scanning electron microscopic evaluation of human dentinal tubules according to age and location. J Endod 1984;10(8):359-363. DOI: 10.1016/S0099-2399(84)80155-7.

28. Almohaimede A, Almanie D, Alaathy $S$, et al. Fracture Resistance of Roots Filled with Bio-Ceramic and Epoxy resin-based sealers: in vitro study. Eur Endod J 2020;5(2):134-137. DOI: 10.14744/eej.2019. 33042. 